\title{
A three-stage dispersion and dissipation of order infinity Runge-Kutta-Nyström method for periodic IVPs
}

\begin{abstract}
In this paper, a new three-stage Runge-Kutta-Nyström (RKN) method with two variable coefficients is constructed. The new method is based on Garcia's RKN method of algebraic order four. By using the idea of dispersion and dissipation of order infinity, a new method for solving second-order ordinary differential equations (ODE) with periodic solutions is derived. Numerical tests show that the new method is more accurate than the other existing methods that were derived in the past.
\end{abstract}

Keyword: Dispersion; Dissipation; Runge-Kutta-Nystrom 